\title{
ITERATIVE PROCESSES WITH ERRORS FOR NONLINEAR EQUATIONS
}

\author{
EJUBomir Ćirić and JeONG Sheok UME
}

\begin{abstract}
In this paper we introduce and consider a class of multi-valued and single-valued operators of generalised monotone type. We proved a new general lemma on the convergence of real sequences and some new convergence theorems for the Ishikawa and Mann iteration processes with errors to the unique fixed point of such operators, which are not necessarily Lipschitz operators. Our results generalise, improve, and extend several recent results.
\end{abstract}

\section{INTRODUCTION}

Let $X$ be a real normed linear space. A mapping $T$ with domain $D(T)$ and range $R(T)$ in $X$ is called accretive ([1]) if for each $x, y$ in $D(T)$ and all $t \geqslant 0$, the following inequality is satisfied:

$$
\|x-y\| \leqslant\|x-y+t(T x-T y)\| .
$$

If $X$ is a Hilbert space, the accretive condition reduces to

$$
\langle T x-T y, x-y\rangle \geqslant 0
$$

for all $x, y \in X$. An early fundamental result in the theory of accretive operators, due to Browder, states that the initial value problem

$$
\frac{d u}{d t}+T u=0, \quad u(0)=u_{0}
$$

is solvable if $T$ is locally Lipschitzian and accretive on $X$. It is well known (see, for example [19]) that many physically significant problems can be modelled in the form of this equation, where $T$ is accretive. Typical examples of how such equations arise are found in models involving either the heat, wave or Schrödinger equation. Browder also proved that if $T: X \rightarrow X$ is locally Lipschitzian and accretive then $T$ is $m$-accretive; that is, the map $(I+T)$, where $I$ denotes the identity map of $X$, is surjective. This result was subsequently generalised by Martin [14] to continuous accretive operators.

Received 3rd March, 2003

This work was supported by Korea Research Foundation Grant (KRF-2003-015-C00039).

Copyright Clearance Centre, Inc. Serial-fee code: 0004-9727/04 \$A2.00+0.00. 
Let $X$ be a Banach space, $X^{*}$ be the dual space of $X$, and $\langle\cdot, \cdot\rangle$ be the pairing betwen $X$ and $X^{*}$. The mapping $J: X \rightarrow 2^{X^{*}}$ defined by

$$
J(x)=\left\{f^{*} \in X^{*}:\left\langle x, f^{*}\right\rangle=\|x\|^{2},\left\|f^{*}\right\|=\|x\|\right\}, \quad x \in X,
$$

is called the normalised duality mapping. Recall (see [12]) that a multi-valued operator $T$ with domain $D(T) \subset X$ and range $R(T) \subset 2^{X}$ is said to be of monotone type if there exist a constant $k \in(0,1)$ and an $x^{*} \in D(T)$ such that for each $x \in D(T)$ there exists $j\left(x-x^{*}\right) \in J\left(x-x^{*}\right)$ satisfying

$$
\left\langle\bar{x}-x^{*}, j\left(x-x^{*}\right)\right\rangle \leqslant k\left\|x-x^{*}\right\|^{2} \quad \text { for each } \bar{x} \in T x .
$$

Also, $T$ is said to be strongly accretive if there exists a constant $k \in(0,1)$ such that for all $x, y \in D(T)$ there exists $j(x-y) \in J(x-y)$ with

$$
\langle\bar{x}-\bar{y}, j(x-y)\rangle \geqslant k\|x-y\|^{2} \quad \text { for each } \quad \bar{x} \in T x \quad \text { and } \quad \bar{y} \in T y .
$$

Finally, $T$ is said to be strongly pseudo-contractive if $(I-T)$ is strongly accretive, where $I$ is the identity operator on $D(T)$. Thus, $T$ is strongly pseudo-contractive if there exists a constant $k \in(0,1)$ such that for each $x, y \in D(T)$ there exists $j(x-y) \in J(x-y)$ such that

$$
\langle\bar{x}-\bar{y}, j(x-y)\rangle \leqslant\|x-y\|^{2}-k\|x-y\|^{2} \quad \text { for each } \bar{x} \in T x \text { and } \bar{y} \in T y \text {. }
$$

The class of operators of monotone type and classes of strongly accretive and strongly pseudo-contractive operators have been studied extensively by various authors. In 1996 Chidume [9] and Osilike [17] introduced and studied a class of $\phi$-strongly accretive and a class of $\phi$-strongly pseudo-contractive operators, which are generalisations of the class of strongly accretive and the class of strongly pseudo-contractive operators.

DEFinition 1.1: $([\mathbf{9}, \mathbf{1 7}])$. An operator $T: D(T) \subset X \rightarrow X$ is said to be $\phi$ strongly accretive if there exists a strictly increasing function $\phi:[0,+\infty) \rightarrow[0,+\infty)$ with $\phi(0)=0$ such that for each $x, y \in D(T)$ there is $j(x-y) \in J(x-y)$ (where $J$ is a normalised duality mapping) such that

$$
\langle T x-T y, j(x-y)\rangle \geqslant \phi(\|x-y\|)\|x-y\| .
$$

If $I-T$ is $\phi$-strongly accretive, that is, if

$$
\langle T x-T y, j(x-y)\rangle \leqslant\|x-y\|^{2}-\phi(\|x-y\|)\|x-y\|,
$$

then $T$ is said to be $\phi$-strongly pseudo-contractive. If $T$ satisfies (1.5) for any $x \in D(T)$, but $y \in F(T)=\{x \in D(T): x \in T x\}$, when $F(T) \neq \emptyset$, then $T$ is said to be $\phi$ hemicontractive.

Now we shall introduce a class of operators of generalised monotone type, a class of generalised strongly accretive and a class of generalised strongly pseudo-contractive and hemicontractive operators. 
Let $X$ be a Banach space and $T: D(T) \subset X \rightarrow 2^{X}$ be a multi-valued operator.

DeFinition 1.2: An operator $T$ is said to be of generalised monotone type if there exists an $x^{*} \in D(T)$ and a nondecreasing function $\varphi:[0,+\infty) \rightarrow[0,+\infty)$ with $\varphi(t)=0$ if and only if $t=0$, such that for each $x \in D(T)$ there exists $j_{p}\left(x-x^{*}\right) \in J_{p}\left(x-x^{*}\right)$; $1<p<+\infty$, such that

$$
\left\langle\bar{x}-x^{*}, j_{p}\left(x-x^{*}\right)\right\rangle \leqslant\left\|x-x^{*}\right\|^{p}-\varphi\left(\left\|x-x^{*}\right\|\right) \text { for each } \bar{x} \in T x,
$$

where $J_{p}$ is the generalised duality map given by

$$
J_{p}(x)=\left\{f^{*} \in X^{*}:\left\langle x, f^{*}\right\rangle=\|x\|^{p},\left\|f^{*}\right\|=\|x\|^{p-1}\right\}, \quad x \in X .
$$

Definition 1.3: An operator $T$ is said to be generalised strongly accretive if for each $x, y \in D(T)$

$$
\left\langle\bar{x}-\bar{y}, j_{p}(x-y)\right\rangle \geqslant \varphi(\|x-y\|) \text { for each } \bar{x} \in T x \text { and } \bar{y} \in T y,
$$

where $j_{p}$ and $\varphi$ are as in Definition 1.2.

DEFINITION 1.4: An operator $T$ is said to be generalised strongly pseudo-contractive if $I-T$ is generalised strongly accretive, that is, if

$$
\left\langle\bar{x}-\bar{y}, j_{p}(x-y)\right\rangle \leqslant\|x-y\|^{p}-\varphi(\|x-y\|) \quad \text { for each } \bar{x} \in T x \text { and } \bar{y} \in T y,
$$

where $j_{p}$ and $\varphi$ are as in Definition 1.2. If $F(T)=\{x \in D(T): x \in T x\}$ is non-empty and if $T$ satisfies (1.8) for all $x \in D(T)$, but $y \in F(T)$, then $T$ is said to be a generalised hemicontractive operator.

Observe that every mapping of monotone type is a mapping of generalised monotone type with $\varphi(t)=(1-k) t^{2}$ and $p=2$. The following example shows that a class of mappings of generalised monotone type is a genuine generalisation of a class of mappings of monotone type.

EXAMPLE 1. Let $X=R$ be the set of real numbers with usual norm, $D=[0,+\infty)$ and $T: D \rightarrow D$ be a single-valued mapping defined by

$$
T x=\frac{x^{3}}{1+x^{2}} .
$$

It is easy to see that $T$ is of generalised monotone type which satisfies (1.6) with $x^{*}=0$, $\varphi(t)=\left(t^{p}\right) /\left(1+t^{p}\right)$. However, $T$ is not of monotone type, since for any $k \in(0,1)$ and $x>\sqrt{k /(1-k)}$ we have

$$
\left\langle\frac{x^{3}}{1+x^{2}}, j(x)\right\rangle=\frac{x^{2}}{1+x^{2}} x^{2}>k \cdot x^{2} .
$$

Observe that $T$ is a generalised hemicontractive, but not $\phi$-hemicontractive operator. Indeed, if we suppose that $T$ satisfies (1.5) with $y=0=T(0)$ and with some function 
$\phi(t)$, where $\phi:[0,+\infty) \rightarrow[0,+\infty)$ is strictly increasing with $\phi(0)=0$, then we get the inequality $\phi(x) \leqslant x /\left(1+x^{2}\right)$ for all $x \in(0,+\infty)$. Thus we have $\lim _{x \rightarrow+\infty} \phi(x)=0$, which is in contradiction with hypothesis that $\phi(0)=0$ and $\phi(x)$ is strictly increasing.

An effective method for approximating the unique fixed point of an operator of monotone type $T: D(T) \subset X \rightarrow 2^{X}$, where $X$ is a Banach space, is the Ishikawa iteration process, starting with an arbitrary $x_{0} \in D(T)$ and for $n \geqslant 0$

$$
x_{n+1} \in\left(1-\alpha_{n}\right) x_{n}+\alpha_{n} T y_{n}, y_{n} \in\left(1-\beta_{n}\right) x_{n}+\beta_{n} T x_{n},
$$

where $\alpha_{n}, \beta_{n} \in[0,1]$ satisfy suitable conditions. This successive process is more useful if it allows nonzero error terms. Such processes are specially useful in the numerical analysis, since in a general case it is more difficult to determine a succesive approximation $x_{n+1}$ exactly from the value of $x_{n}$, than an approximative. Recently Liu [13] introduced the Ishikawa iteration process with errors for an operator $T: D(T) \subset X \rightarrow X$. Starting with $x_{0} \in D(T)$ and for $n \geqslant 0$

$$
\begin{aligned}
x_{n+1} & =\left(1-\alpha_{n}\right) x_{n}+\alpha_{n} T y_{n}+u_{n}, \\
y_{n} & =\left(1-\beta_{n}\right) y_{n}+\beta_{n} T x_{n}+v_{n},
\end{aligned}
$$

where $\left.\left\{\alpha_{n}\right\}, \beta_{n}\right\} \subset[0,1]$ and $\left\{u_{n}\right\},\left\{v_{n}\right\} \subset X$ are two summable sequences (that is, $\left.\sum_{n=0}^{\infty}\left\|u_{n}\right\|<+\infty, \sum_{n=0}^{\infty}\left\|v_{n}\right\|<+\infty\right)$. If $\beta_{n}=0$ and $v_{n}=0$, then the sequence $\left\{x_{n}\right\}$ is called the Mann iteration process with errors.

The purpose of this paper is to consider a class of operators of generalised monotne type, defined by Definition 1.2, and to study the Ishikawa iteration process with errors, where error terms $\left\{u_{n}\right\},\left\{v_{n}\right\} \subset X$ are not necessarily summable. Our results on the convergence of the iterative approximation of solutions to nonlinear equations of generalised monotone type in arbitrary Banach spaces generalise corresponding resultus of Gu Feng [12] and generalise, improve and extend many known results.

\section{LEMMAS AND INEQUALITIES}

LEMMA 2.1. Let $\left\{\rho_{n}\right\}_{n=0}^{\infty}$ be a sequence of non-negative real numbers, $\left\{\lambda_{n}\right\}$ be a real sequence satisfying

$$
0 \leqslant \lambda_{n} \leqslant 1 \text { and } \sum_{n=0}^{\infty} \lambda_{n}=+\infty
$$

and let $\varphi:[0,+\infty) \rightarrow[0,+\infty)$ be a non-decreasing real function such that $\varphi(t)=0$ if and only if $t=0$. If there exists a positive integer $k$ such that

$$
\rho_{n+1}^{p} \leqslant \rho_{n}^{p}-\lambda_{n} \varphi\left(\rho_{n+1}\right)+\lambda_{n} \mu_{n}
$$


for all $n \geqslant k$, where $p>0$ and $\mu_{n} \geqslant 0$ with $\lim \mu_{n}=0$, then

$$
\lim _{n \rightarrow \infty} \rho_{n}=0
$$

ProOF: First we show that

$$
2 \delta=\lim _{n \rightarrow \infty} \inf \rho_{n}=0 .
$$


We show that (2.7) implies that $\rho_{s} \geqslant \rho_{s+1}$. From (2.7), (2.2) and (2.5) we get, as $\lambda_{s} \leqslant 1$,

$$
2 \varepsilon^{p} \leqslant \rho_{s+1}^{p} \leqslant \rho_{s}^{p}-\lambda_{s} \varphi\left(\rho_{s+1}\right)+\lambda_{s} \cdot \mu_{s} \leqslant \rho_{s}^{p}+\lambda_{s} \mu_{s} \leqslant \rho_{s}^{p}+\mu_{s} \leqslant \rho_{s}^{p}+\varepsilon^{p} .
$$

Hence we get $\rho_{s} \geqslant \varepsilon$. Since from (2.7), $\rho_{s+1} \geqslant \varepsilon$ and as $\varphi(t)$ is non-decreasing, we have $-\varphi\left(\rho_{s+1}\right) \leqslant-\varphi(\varepsilon)$. Thus from (2.2) and (2.5) we obtain

$$
\rho_{s+1}^{p} \leqslant \rho_{s}^{p}-\lambda_{s} \varphi\left(\rho_{s+1}\right)+\lambda_{s} \mu_{s} \leqslant \rho_{s}^{p}-\lambda_{s} \varphi(\varepsilon)+\lambda_{s} \varphi(\varepsilon)=\rho_{s}^{p} .
$$

Thus we showed that $\rho_{s+1} \leqslant \rho_{s}$.

Similarly, since $\rho_{s} \geqslant \rho_{s+1}$ and (2.7) imply that $\rho_{s}^{p} \geqslant 2 \varepsilon^{p}$, we can show that $\rho_{s} \leqslant \rho_{s-1}$. Continuing this process we obtain

$$
\rho_{s+1} \leqslant \rho_{s} \leqslant \rho_{s-1} \ldots \leqslant \rho_{r}
$$

Now, by (2.6) and (2.7), we have $2 \varepsilon^{p} \leqslant \rho_{s+1}^{p} \leqslant \rho_{r}^{p}<\varepsilon^{p}$, a contradiction. Thus (2.4) is wrong. Therefore, we have

$$
\lim _{n \rightarrow \infty} \sup \rho_{n}=0
$$

which implies that $\lim _{n \rightarrow \infty} \rho_{n}=0$.

LEmma 2.2. (Chang, Cho, Lee, Jung and Kang [3.0f Let $X$ be a real Banach space and let $J_{p}: X \rightarrow 2^{X^{*}}, 1<p<+\infty$, be a generalised duality mapping. Then, for any given $x, y \in X$, we have

$$
\|x+y\|^{p} \leqslant\|x\|^{p}+p\left\langle y, j_{p}(x+y)\right\rangle
$$

for all $j_{p}(x+y) \in J_{p}(x+y)$.

\section{THE CONVERGENCE OF ISHIKAWA ITERATION SEQUENCES FOR OPERATORS OF GENERALISED MONOTONE TYPE}

THEOREM 3.1. Let $D$ be a nonempty subset of a Banach space $X$ and $T: D$ $\rightarrow 2^{X}$ be a multi-valued operator of generalised monotone type such that $T(D)$ is bounded and $F(T)=\{x \in D: x \in T x\}$ is non-empty. Let $\left\{\alpha_{n}\right\}$ and $\left\{\beta_{n}\right\}$ be real sequences in $[0,1]$ and $\left\{u_{n}\right\}$ and $\left\{v_{n}\right\}$ be sequences in $X$ satisfying the following conditions:

$$
\begin{aligned}
& \text { (i) } \alpha_{n} \rightarrow 0 \text { as } n \rightarrow \infty \text { and } \sum_{n=1}^{\infty} \alpha_{n}=+\infty \text {, } \\
& \text { (ii) }\left\|u_{n}\right\|=o\left(\alpha_{n}\right) \text {. }
\end{aligned}
$$

Suppose that there exists $x_{0} \in D$ such that Ishikawa iteration process with errors $\left\{x_{n}\right\}$, $\left\{y_{n}\right\}$ generated from $x_{0}$ by

$$
\begin{aligned}
x_{n+1} & =\left(1-\alpha_{n}\right) x_{n}+\alpha_{n} \bar{y}_{n}+u_{n} ; \quad \bar{y}_{n} \in T y_{n}, \\
y_{n} & =\left(1-\beta_{n}\right) x_{n}+\beta_{n} \bar{x}_{n}+v_{n} ; \quad \bar{x}_{n} \in T x_{n}, n \geqslant 0
\end{aligned}
$$

is included in $D$ and sequences $\left\{\bar{x}_{n}\right\}$ and $\left\{\bar{y}_{n}\right\}$ satisfy the following condition: 
(iii) $\left\|\bar{x}_{n+1}-\bar{y}_{n}\right\| \rightarrow 0$ as $n \rightarrow \infty$.

Then the Ishikawa iteration sequence $\left\{x_{n}\right\}$ with errors converges strongly to the unique fixed point $x^{*}$ of $T$, where $x^{*} \in D$ is the point appearing in (1.6).

ProOF: Set

$$
\begin{aligned}
\omega_{n} & =\frac{1}{\alpha_{n}} u_{n}, \quad \text { if } \alpha_{n} \neq 0 \\
& =0, \quad \text { if } \alpha_{n}=0
\end{aligned}
$$

Since $T(D)$ is bounded, by (ii) we have that

$$
M=\left\|x_{0}-x^{*}\right\|+\sup \left\{\left\|\bar{x}-x^{*}\right\|: \bar{x} \in T x, x \in D\right\}+\sup \left\{\left\|\omega_{n}\right\|: n \geqslant 0\right\}<+\infty .
$$

No we shall show that

$$
\left\|x_{n}-x^{*}\right\| \leqslant M \text { for all } n \geqslant 0 .
$$

We prove (3.4) by induction. For $n=0,(3.4)$ holds from (3.3). Suppose now that (3.4) is true for some fixed $n \geqslant 0$. From (3.1) we have

$$
\begin{aligned}
\left\|x_{n+1}-x^{*}\right\| & =\left\|\left(1-\alpha_{n}\right)\left(x_{n}-x^{*}\right)+\alpha_{n}\left(\bar{y}_{n}-x^{*}\right)+u_{n}\right\| \\
& \leqslant\left(1-\alpha_{n}\right)\left\|x_{n}-x^{*}\right\|+\alpha_{n}\left\|\bar{y}_{n}-x^{*}\right\|+\alpha_{n}\left\|\omega_{n}\right\| .
\end{aligned}
$$

Thus, by (3.3), we get

$$
\left\|x_{n+1}-x^{*}\right\| \leqslant\left(1-\alpha_{n}\right)\left\|x_{n}-x^{*}\right\|+\alpha_{n} M .
$$

Now, by the induction hypothesis $\left\|x_{n}-x^{*}\right\| \leqslant M$, we obtain $\left\|x_{n+1}-x^{*}\right\| \leqslant\left(1-\alpha_{n}\right) M$ $+\alpha_{n} M=M$. Therefore, we proved (3.4).

Since a real function $f:[0,+\infty) \rightarrow[0,+\infty)$, defined by $f(t)=t^{p}, 1<p<+\infty$, is increasing and convex, that is, for any $\lambda \in[0,1]$ and $t_{1}, t_{2}>0$, we have that

$$
\left((1-\lambda) t_{1}+\lambda t_{2}\right)^{p} \leqslant(1-\lambda) t_{1}^{p}+\lambda t_{2}^{p}
$$

from (3.5) we get

$$
\begin{aligned}
\left\|x_{n+1}-x^{*}\right\|^{p} & \leqslant\left(\left(1-\alpha_{n}\right)\left\|x_{n}-x^{*}\right\|+\alpha_{n} M\right)^{p} \\
& \leqslant\left(1-\alpha_{n}\right)\left\|x_{n}-x^{*}\right\|^{p}+\alpha_{n} M^{p}
\end{aligned}
$$

Thus we obtain, as $1-\alpha_{n} \leqslant 1$,

$$
\left\|x_{n+1}-x^{*}\right\|^{p} \leqslant\left\|x_{n}-x^{*}\right\|^{p}+\alpha_{n} M^{p} .
$$


From Lemma 2.2 and (3.1) we have

$$
\begin{aligned}
\left\|x_{n+1}-x^{*}\right\|^{p}= & \left\|\left(1-\alpha_{n}\right)\left(x_{n}-x^{*}\right)+\alpha_{n}\left(\bar{y}_{n}-x^{*}\right)+u_{n}\right\|^{p} \\
\leqslant & \left(1-\alpha_{n}\right)^{p}\left\|x_{n}-x^{*}\right\|^{p}+p \alpha_{n}\left\langle\bar{y}_{n}-x^{*}, j_{p}\left(x_{n+1}-x^{*}\right)\right\rangle \\
\quad & \quad+p\left\langle u_{n}, j_{p}\left(x_{n+1}-x^{*}\right)\right\rangle \\
= & \left(1-\alpha_{n}\right)^{p}\left\|x_{n}-x^{*}\right\|^{p}+p \alpha_{n}\left\langle\bar{x}_{n+1}-x^{*}, j_{p}\left(x_{n+1}-x^{*}\right)\right\rangle \\
& \quad+p \alpha_{n}\left\langle\bar{y}_{n}-\bar{x}_{n+1}, j_{p}\left(x_{n+1}-x^{*}\right)\right\rangle+p \alpha_{n}\left\langle w_{n}, j_{p}\left(x_{n+1}-x^{*}\right)\right\rangle
\end{aligned}
$$

for all $j_{p}\left(x_{n+1}-x^{*}\right) \in J_{p}\left(x_{n+1}-x^{*}\right)$. Hence we get, as $T$ is of generalised monotone type,

$$
\begin{aligned}
\left\|x_{n+1}-x^{*}\right\|^{p} \leqslant\left(1-\alpha_{n}\right)^{p}\left\|x_{n}-x^{*}\right\|^{p}+p \alpha_{n}\left\|x_{n+1}-x^{*}\right\|^{p} & \\
& -p \alpha_{n} \varphi\left(\left\|x_{n+1}-x^{*}\right\|\right)+p \alpha_{n}\left(b_{n}+c_{n}\right)
\end{aligned}
$$

where

$$
\begin{aligned}
& b_{n}=\left\langle\bar{y}_{n}-\bar{x}_{n+1}, j_{p}\left(x_{n+1}-x^{*}\right)\right\rangle \\
& c_{n}=\left\langle\omega_{n}, j_{p}\left(x_{n+1}-x^{*}\right)\right\rangle .
\end{aligned}
$$

Since a generalised duality mapping $J_{p}$ on a bounded subset of $X$ is a bounded subset in $X^{*}$, and as $\left\|x_{n+1}-x^{*}\right\| \leqslant M$, it follows that $\left\{j_{p}\left(x_{n+1}-x^{*}\right)\right\}$ is a bounded subset in $X^{*}$. Thus, from (iii) we have that

$$
\lim _{n \rightarrow \infty} b_{n}=0,
$$

and from (ii), because $\left\|\omega_{n}\right\|=\left(1 / \alpha_{n}\right)\left\|u_{n}\right\| \rightarrow 0$ as $n \rightarrow \infty$,

$$
\lim _{n \rightarrow \infty} c_{n}=0 .
$$

Now we shall estimate $\left(1-\alpha_{n}\right)^{p}$. Consider the exponential function $f(x)=e^{-x}$ for $x \geqslant 0$. We know that for each $x>0$ there exists $c=c(x) \in(0, x)$ such that

$$
f(x)=f(0)+f^{\prime}(0) x+\frac{1}{2} f^{\prime \prime}(c) x^{2} .
$$

Since $f^{\prime \prime}(c)=e^{-c}$ and as $0<e^{-c}<1$, we obtain

$$
f(0)+f^{\prime}(0) x<f(x)<f(0)+f^{\prime}(0) x+\frac{1}{2} x^{2} .
$$

Thus we have

$$
1-x \leqslant e^{-x} \leqslant 1-x+\frac{1}{2} x^{2} \text { for all } x \geqslant 0 .
$$

Since $0 \leqslant 1-\alpha_{n} \leqslant e^{-\alpha_{n}}$ implies $\left(1-\alpha_{n}\right)^{p} \leqslant e^{-p \alpha_{n}}$ for any $p>0$, and as by (3.10) we have that $e^{-p \alpha_{n}} \leqslant 1-p \alpha_{n}+\left(p^{2} / 2\right) \alpha_{n}^{2}$, we obtain

$$
\left(1-\alpha_{n}\right)^{p} \leqslant 1-p \alpha_{n}+\left(p^{2} / 2\right) \alpha_{n}^{2} \text {. }
$$


Using (3.11), (3.6) and (3.4) we get, as $p>1$,

$$
\begin{aligned}
\left(1-\alpha_{n}\right)^{p} \| x_{n} & -x^{*}\left\|^{p}+p \alpha_{n}\right\| x_{n+1}-x^{*} \|^{p} \\
& \leqslant\left(1-\alpha_{n}\right)^{p}\left\|x_{n}-x^{*}\right\|^{p}+p \alpha_{n}\left(\left\|x_{n}-x^{*}\right\|^{p}+\alpha_{n} M^{p}\right) \\
& \leqslant\left(1+\frac{1}{2} p^{2} \alpha_{n}^{2}\right)\left\|x_{n}-x^{*}\right\|^{p}+p \alpha_{n}^{2} M^{p} \\
& \leqslant\left\|x_{n}-x^{*}\right\|^{p}+2 p^{2} \alpha_{n}^{2} M^{p}
\end{aligned}
$$

Substituting (3.12) into (3.7) we get

$$
\left\|x_{n+1}-x^{*}\right\|^{p} \leqslant\left\|x_{n}-x^{*}\right\|^{p}+2 p^{2} \alpha_{n}^{2} M^{p}-p \alpha_{n} \varphi\left(\left\|x_{n+1}-x^{*}\right\|\right)+p \alpha_{n}\left(b_{n}+c_{n}\right) .
$$

Hence we obtain, as $p>1$,

$$
\left\|x_{n+1}-x^{*}\right\|^{p} \leqslant\left\|x_{n}-x^{*}\right\|^{p}-\alpha_{n} \varphi\left(\left\|x_{n+1}-x^{*}\right\|\right)+\alpha_{n} \mu_{n},
$$

where

$$
\mu_{n}=p\left(2 p \alpha_{n} M^{p}+b_{n}+c_{n}\right) \rightarrow 0 \text { as } n \rightarrow \infty \text {. }
$$

Letting $\rho_{n}=\left\|x_{n}-x^{*}\right\|$ and $\lambda_{n}=\alpha_{n}$, it follows from (3.13) and Lemma 2.1 that $\| x_{n}$ $-x^{*} \| \rightarrow 0$ as $n \rightarrow \infty$. Thus we proved that $\left\{x_{n}\right\}$ converges strongly to the point $x^{*} \in D$.

Now we show that $x^{*}$ is a unique fixed point of $T$. Let $q \in F(T)$. Since $T$ is of generalised monotone type and $q \in T q$, from (1.6) with $\bar{x}=q$ we have

$$
\left\|q-x^{*}\right\|^{p}=\left\langle\bar{q}-x^{*}, j_{p}\left(q-x^{*}\right)\right\rangle \leqslant\left\|q-x^{*}\right\|^{p}-\varphi\left(\left\|q-x^{*}\right\|\right) .
$$

Thus we have, as $\varphi(t) \in[0,+\infty)$,

$$
0 \leqslant \varphi\left(\left\|q-x^{*}\right\|\right) \leqslant 0
$$

Since $\varphi(t)=0$ if and only if $t=0$, we have $\left\|q-x^{*}\right\|=0$; hence $q=x^{*}$. Therefore, $x^{*}$ is the unique fixed point of $T$ and $\left\{x_{n}\right\}$ converges strongly to the point $x^{*}$.

REMARK 3.2. From Definition 1.4 it is easy to see that a strongly pseudo-contractive operator with a fixed point is an operator of generalised monotone type which satisfies (1.6) with $p=2$ and $\varphi(t)=k t^{2}$.

REMARK 3.3. Theorem 3.1 improves and extends the corresponding results of Gu Feng [12, Theorem 2.1] and Chang and Tan [2, Theorem 3.1] and contain it as special cases. We note that $\left\{\left\|u_{n}\right\|\right\}$ is not necessarily summable. Prototypical $\alpha_{n}$ and upper bound for $\left\|u_{n}\right\|$ can be

$$
\alpha_{n}=\frac{1}{\sqrt{1+n}}, \quad\left\|u_{n}\right\| \leqslant \frac{1}{1+n} .
$$

Now we state the following defintion. 
Definition 3.1: A multi-valued mapping $T: D \subset X \rightarrow 2^{X}$ is said to be uniformly continuous on a subset $A$ of $D$, if for any $\varepsilon>0$ there exists a $\eta>0$ such that

$$
\delta(T(x), T(y))=\sup \{\|\bar{x}-\bar{y}\|: \bar{x} \in T(x), \bar{y} \in T(y)\}<\varepsilon
$$

whenever $\|x-y\|<\eta ; x, y \in A$.

Corollary 3.1. Let $D$ be a nonempty subset of a Banach space $X$ and $T$ : $D \rightarrow 2^{X}$ be a multi-valued uniformly continuous operator of generalised monotone type such that $T(D)$ is bounded and $F(T)=\{x \in D: x \in T x\}$ is non-empty. Let $\left\{\alpha_{n}\right\}$ and $\left\{\beta_{n}\right\}$ be real sequences in $[0,1]$ and $\left\{u_{n}\right\},\left\{v_{n}\right\}$ be sequences in $X$ satisfying the following conditions:

(i) $\alpha_{n} \rightarrow 0, \beta_{n} \rightarrow 0$ as $n \rightarrow \infty$ and $\sum_{n=1}^{\infty} \alpha_{n}=+\infty$,

(ii) $\left\|u_{n}\right\|=o\left(\alpha_{n}\right)$,

(iii) $\left\|v_{n}\right\| \rightarrow 0$ as $n \rightarrow \infty$.

Suppose that there exists $x_{0} \in D$ such that the Ishikawa iteration process with errors $\left\{x_{n}\right\},\left\{y_{n}\right\}$ generated from $x_{0}$ by

$$
\begin{aligned}
x_{n+1} & =\left(1-\alpha_{n}\right) x_{n}+\alpha_{n} \bar{y}_{n}+u_{n} ; \quad \bar{y}_{n} \in T y_{n}, \\
y_{n} & =\left(1-\beta_{n}\right) x_{n}+\beta_{n} \bar{x}_{n}+v_{n} ; \quad \bar{x}_{n} \in T x_{n}, n \geqslant 0
\end{aligned}
$$

is included in $D$. Then the Ishikawa iteration sequence $\left\{x_{n}\right\}$ with errors converges strongly to the unique fixed point $x^{*}$ of $T$, where $x^{*} \in D$ is the point appearing in (1.6).

PROOF: It is sufficient to show that the condition (iii) in Theorem 3.1 is satisfied.

From definition of $x_{n+1}$ and $y_{n}$ we have

$$
x_{n+1}-y_{n}=\left(\beta_{n}-\alpha_{n}\right) x_{n}+\alpha_{n} T y_{n}-\beta_{n} T x_{n}+u_{n}-v_{n}
$$

From hypotheses of Theorem, $\left\{T y_{n}\right\},\left\{T x_{n}\right\},\left\{u_{n}\right\}$ and $\left\{v_{n}\right\}$ are bounded sequences. Since by the triangle inequeality we have $\left\|x_{n}\right\| \leqslant\left\|x_{n}-x^{*}\right\|+\left\|x^{*}\right\|$, following lines in the proof of Theorem 3.1 we can prove that $\left\{x_{n}\right\}$ is bounded. Thus, from (ii), (iii) and (3.16) it follows that

$$
\left\|x_{n+1}-y_{n}\right\| \rightarrow 0 \text { as } n \rightarrow \infty
$$

By virtue of the uniform continuity of $T$, we have

$$
\left\|\bar{x}_{n+1}-\bar{y}_{n}\right\| \rightarrow 0 \text { as } n \rightarrow \infty \text {. }
$$

Thus the condition (iii) of Theorem 3.1 is satisfied.

COROLlary 3.2. Let $D$ be a nonempty subset of a Banach space $X$ and $T$ : $D \rightarrow 2^{X}$ be a multi-valued operator of generalised monotone type such that $T(D)$ is bounded and $F(T)=\{x \in D: x \in T x\}$ is non-empty. Let $\left\{\alpha_{n}\right\}$ and $\left\{\beta_{n}\right\}$ be real sequences in $[0,1]$ satisfying the following conditions: 
(i) $\alpha_{n} \rightarrow 0$ as $n \rightarrow \infty$ and $\sum_{n=1}^{\infty} \alpha_{n}=+\infty$.

Suppose that there exists $x_{0} \in D$ such that the Ishikawa iteration process $\left\{x_{n}\right\},\left\{y_{n}\right\}$ generated from $x_{0}$ by

$$
\begin{aligned}
x_{n+1}=\left(1-\alpha_{n}\right) x_{n}+\alpha_{n} \bar{y}_{n} ; \quad \bar{y}_{n} \in T y_{n}, \\
y_{n}=\left(1-\beta_{n}\right) x_{n}+\beta_{n} \bar{x}_{n} ; \quad \bar{x}_{n} \in T x_{n}, n \geqslant 0,
\end{aligned}
$$

is included in $D$ and sequences $\left\{\bar{x}_{n}\right\}$ and $\left\{\bar{y}_{n}\right\}$ satisfy the following condition:

(ii) $\left\|\bar{x}_{n+1}-\bar{y}_{n}\right\| \rightarrow 0$ as $n \rightarrow \infty$.

Then the Ishikawa iteration sequence $\left\{x_{n}\right\}$ converges strongly to the unique fixed point $x^{*}$ of $T$, where $x^{*} \in D$ is the point appearing in (1.6).

Proof: Taking $u_{n}=v_{n}=0$ for all $n \geqslant 0$ in Theorem 3.1, then conclusions of Corollary 3.2 follow from Theorem 3.1 immediately.

Corollary 3.3. Let $D$ be a nonempty subset of a Banach space $X$ and $T$ : $D \rightarrow 2^{X}$ be a multi-valued uniformly continuous operator of generalised monotone type such that $T(D)$ is bounded and $F(T) \neq \emptyset$. Let $\left\{\alpha_{n}\right\}$ and $\left\{\beta_{n}\right\}$ be real sequences in $[0,1]$ satisfying the following conditions:

(i) $\alpha_{n} \rightarrow 0, \beta_{n} \rightarrow 0$ as $n \rightarrow \infty$ and $\sum_{n=1}^{\infty} \alpha_{n}=+\infty$.

Suppose that there exists $x_{0} \in D$ such that the Ishikawa iteration process $\left\{x_{n}\right\},\left\{y_{n}\right\}$ generated from $x_{0}$ by

$$
\begin{aligned}
x_{n+1}=\left(1-\alpha_{n}\right) x_{n}+\alpha_{n} \bar{y}_{n}, \quad \bar{y}_{n} \in T y_{n} \\
y_{n}=\left(1-\beta_{n}\right) x_{n}+\beta_{n} \bar{x}_{n}, \quad \bar{x}_{n} \in T x_{n}, \quad n \geqslant 0
\end{aligned}
$$

is included in $D$. Then the Ishikawa iteration sequence $\left\{x_{n}\right\}$ converges strongly to the unique fixed point $x^{*}$ of $T$, where $x^{*} \in D$ is the point appearing in (1.6).

Proof: Taking $u_{n}=v_{n}=0$ for all $n \geqslant 0$ in Corollary 3.1, then conclusions of Corollary 3.3 follow from Corollary 3.1 immediately.

Remark 3.8. Our results in Theorem 3.1 and Corollaries 3.1-3.3 improve, extend and generalise the corresponding results of Gu Feng [12], Chang and Tan [3], Chidume [5], and Dunn [11]. Moreover, since every strongly pseudo-contractive mapping with fixed point is a special case of mappings of generalised monotone type, Theorem 3.1 and Corollaries 3.1-3.3 also improve, extend and generalise the corresponding results for strongly pseudo contractive mappings of Chang [4], Chidume [7], Chidume [8], Deng and Ding [10], Osilike [16] and Tan and Xu [18]. 


\section{The CONERGENCE OF THE MANN ITERATION SEQUENCE FOR OPERATOR OF GENERALISED MONOTONE TYPE}

THEOREM 4.1. Let $D$ be a nonempty subset of a Banach space $X$ and $T: D$ $\rightarrow 2^{X}$ be a multi-valued operator of generalised monotone type such that $T(D)$ is bounded and $F(T)=\{x \in D: x \in T x\}$ is non-empty. Let $\left\{\alpha_{n}\right\}$ be a real sequence in $[0,1]$ and $\left\{u_{n}\right\}$ be a sequence in $X$ satisfying the following conditions:

(i) $\alpha_{n} \rightarrow 0$ as $n \rightarrow \infty$ and $\sum_{n=1}^{\infty} \alpha_{n}=+\infty$,

(ii) $\left\|u_{n}\right\|=o\left(\alpha_{n}\right)$.

Suppose that there exists $x_{0} \in D$ such that the Mann iteration process with errors $\left\{x_{n}\right\}$ generated from $x_{0}$ by

$$
x_{n+1}=\left(1-\alpha_{n}\right) x_{n}+\alpha_{n} \bar{x}_{n}+u_{n} ; \quad \bar{x}_{n} \in T x_{n}
$$

is included in $D$ and a sequence $\left\{\bar{x}_{n}\right\}$ satisfies the following condition:

(iii) $\left\|\bar{x}_{n+1}-\bar{x}_{n}\right\| \rightarrow 0$ as $n \rightarrow \infty$.

Then the Ishikawa iteration sequence $\left\{x_{n}\right\}$ with errors converges strongly to the unique fixed point $x^{*}$ of $T$, where $x^{*} \in D$ is the point appearing in (1.6).

Proof: Taking $\beta_{n}=0$ and $v_{n}=0$ for all $n \geqslant 0$ in Theorem 3.1, then the conclusions of Theorem 4.1 follow from Theorem 3.1 immediately.

REMARK 4.2. Observe that, similarly as in Theorem 3.1, the hypotheses in Corollary 3.1, Corollary 3.2 and Corollary 3.3 can be adapted to ensure the strong convergence of the Mann iteration sequences.

\section{REFERENCES}

[1] F.E. Browder, 'Nonlinear monotone and accreative operators in Banach spaces', Proc. Nat. Acad. Sci. U.S.A. 61 (1968), 388-393.

[2] S.S. Chang and K.K. Tan, 'Iteration processes for approximating fixed points of operators of monotone type', Bull. Austral. Math. Soc. 57 (1998), 433-445.

[3] S.S. Chang, Y.J. Cho, B.S. Lee, J.S. Jung and S.M. Kang, 'Iterative approximations of fixed points and solutions for strongly accretive and strongly pseudo-contractive mappings in Banach spaces', J. Math. Anal. Appl. 224 (1998), 149-165.

[4] S.S. Chang, 'Some problems and results in the study of nonlinear analysis', Nonlinear Anal. 30 (1997), 4197-4208.

[5] C.E. Chidume, 'Iterative construction of fixed points for multi-valued operators of the monotone type', Appl. Anal. 23 (1986), 209-218.

[6] C.E. Chidume, 'Iterative solution of nonlinear equations of the monotone type in Banach spaces', Bull. Austral. Math. Soc. 42 (1990), 21-31.

[7] C.E. Chidume, 'Approximation of fixed points of strongly pseudo-contractive mappings', Proc. Amer. Math. Soc. 120 (1994), 545-551. 
[8] C.E. Chidume, 'Iterative solution of nonlinear equations with strongly accretive operators', J. Math. Anal. Appl. 192 (1995), 502-518.

[9] C.E.Chidume, 'Steepest descent approximations for accretive operator equations', Nonlinear Anal. 26 (1996), 299-311.

[10] L. Deng and X. P. Ding, 'Iterative approximation of Lipschitz strictly pseudo-contractive mappings in uniformly smooth Banach spaces', Nonlinear Anal. 24 (1995), 981-987.

[11] J.C. Dunn, 'Iterative construction of fixed points for multi-valued operators of the monotone type', J. Funct. Anal. 27 (1978), 38-50.

[12] F. Gu Iteration processes with errors approximating fixed points of operators of monotone type, Proc. Amer. Math. Soc. 129 (2001), 2293-2300 (electronic).

[13] L.S. Liu, 'Ishikawa and Mann iterative process with errors for nonlinear strongly accretive mappings in Banach spaces', J. Math. Anal. Appl. 194 (1995), 114-125.

[14] R.H. Martin, Jr., 'A global existence theorem for autonomous differential equations in Banach spaces', Proc. Amer. Math. Soc. 26 (1970), 307-314.

[15] M.O. Osilike, 'Ishikawa and Mann iteration methods for nonlinear strongly accretive mappings', Bull. Austral. Math. Soc. 46 (1992), 411-422.

[16] M.O. Osilike, 'Stable iteration procedures for strong pseudo-contractions and nonlinear operator equations of the accretive type', J. Math. Anal. Appl. 204 (1996), 677-692.

[17] M.O. Osilike, 'Iterative solution of nonlinear equations of the $\phi$-strongly accretive type', J. Math. Anal. Appl. 200 (1996), 259-271.

[18] K.K. Tan and H.K. Xu, 'Iterative solutions to nonlineear equations of strongly accretive operators in Banach spaces', Math. Anal. Appl. 178 (1993), 9-21.

[19] E. Zeidler, Nonlinear functional analysis and its applications. Part II. Monotone operators (Springer-Verlag, New York, Berlin, Heidelberg, 1985).

Faculty of Mechanical Engineering

University of Belgrade

Belgrade, 27. marta 80

Yugoslavia

e-mail: lciric@mas.bg.ac.yu
Department of Applied Mathematics Changwon National University

Changwon 641-773

Korea

e-mail: jsume@changwon.ac.kr 